\title{
Characterization and mitigation of Laser-Guide-Star-induced aberrations
}

\author{
Olivier Lardière ${ }^{1, a}$, Rodolphe Conan ${ }^{1}$, Colin Bradley ${ }^{1}$, Kate Jackson ${ }^{1}$, and Glen Herriot ${ }^{2}$ \\ 1 AO Laboratory, Mechanical Engineering Department, University of Victoria, BC, Canada \\ 2 NRC-Herzberg Institute of Astrophysics, Victoria, BC, Canada
}

\begin{abstract}
Sodium Laser Guide Stars (LGS) induce optical aberrations in adaptive optics (AO) systems. The artificial star is elongated due to the sodium layer thickness, and the variations of the sodium layer altitude and atom density profile induce errors on centroid measurements of elongated spots. In AO systems, these errors generate spurious optical aberrations, termed LGS aberrations, especially with ELTs for which the spot elongation is greater. According to analytical models and experimental results obtained with the University of Victoria LGS bench demonstrator, we characterized the main LGS aberrations and studied two options to mitigate them: the Radial Thresholding and the temporal filtering.
\end{abstract}

\section{Origins of the LGS-aberrations}

Temporal variations of the sodium layer density profile induce errors on centroids of the elongated LGS spots of Sack-Hartmann wavefront sensors (SH-WFSs) (Fig. 1). If the laser is launched from behind the secondary mirror, the main LGS aberration is a focus error due to the variation of the mean altitude of the sodium layer. This error is real and must be negated optically by zoom optics in order to refocus the LGS spots [1].

According to a model [2] and experimental results [3,4] two main kinds of LGS-aberrations arise beyond the focus: centro-symmetric aberrations $\left(Z_{11}, Z_{22} \ldots\right)$ and square symmetric aberrations $\left(Z_{14}\right.$, $Z_{26}$ ).

Centro-symmetric aberrations are due to:

- a circular truncation of asymmetric spots by a field-stop or a pixel thresholding,

- the variations of the optical aberrations of the LGS path (including zoom optics) with the LGS distance.

Square symmetric aberrations are due to:

- a square truncation of spots by the pixels boundaries.

- a spot sampling by square pixels or quad-cell.

Consequently, all the LGS-aberrations beyond the focus are only instrumental artefacts and must be mitigated. Most of the LGS-aberrations can be virtually cancelled if the LGS-WFS features :

- a large field of view (FOV) per sub-aperture (wide enough to image a 20km-tick sodium profile),

- a threshold-free centroiding algorithm (such as the Matched Filter [5], the Correlation [6]) or a radial-threshold Centre-of-Gravity (CoG) algorithm [4],

- a calibration procedure and a look-up table to negated the variable optical aberrations of the LGS path,

- a polar-coordinate CCD array [6] to mitigate square symmetric aberrations.

If after taking all these precautions, some LGS-aberrations are still arising, the ultimate solution consists in filtering out the residual aberrations in the temporal frequency domain with the use of a low-bandwidth natural guide star (NGS) WFS.

Section 2 describes the radial-threshold Centre-of-Gravity (CoG) algorithm, while section 3 deals with the filtering of LGS aberrations in the frequency domain.

${ }^{\text {a }}$ e-mail: lardiere@uvic.ca

This is an Open Access article distributed under the terms of the Creative Commons Attribution-Noncommercial License, which permits unrestricted use, distribution, and reproduction in any noncommercial medium, provided the original work is properly cited. 

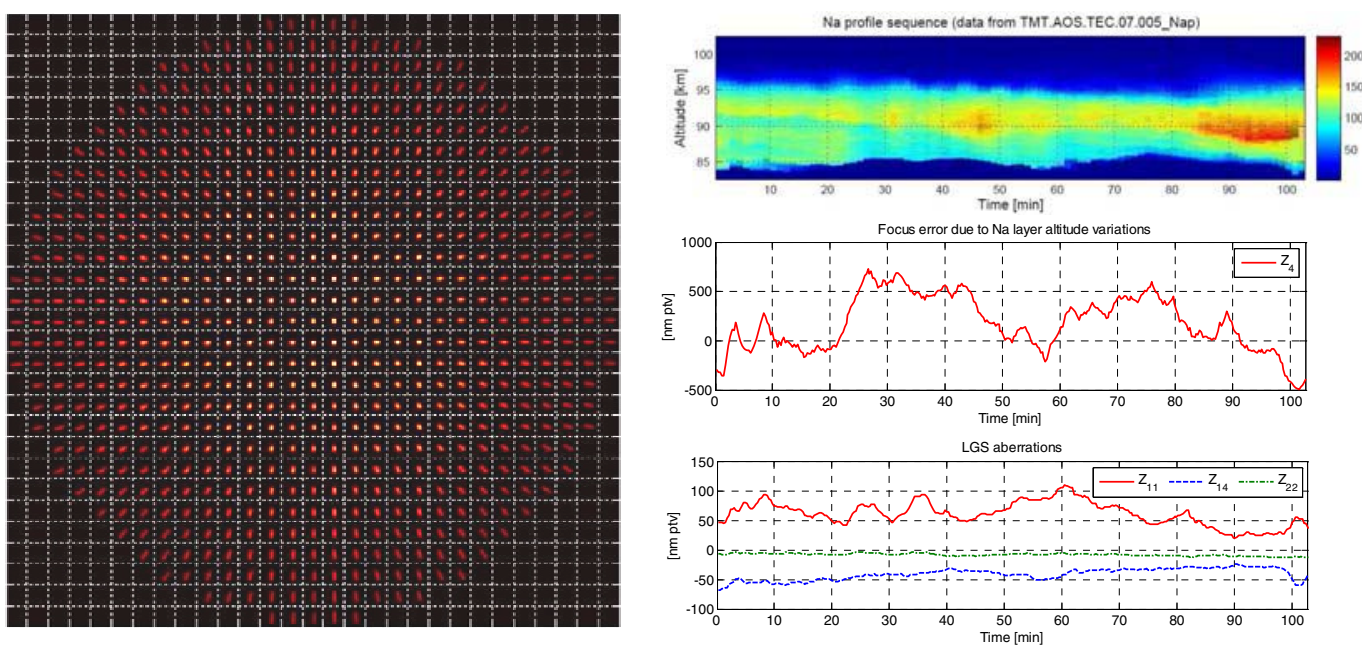

Fig. 1. Elongated LGS spot image and LGS-aberrations induced by the fluctuation of the sodium layer (time series of 88 sodium profiles from the LIDAR of University of Western Ontario).

\section{Radial thresholding}

For CoG-based SH-WFS, a uniform threshold is usually applied on the pixels of the image prior to computing the spot centroids in order to discard the contribution of the background. However, the thresholding also discards the two fainter extremities of each elongated spot and may act as a circular field-stop, generating centro-symmetric LGS aberrations. This statement is confirmed by an analytical model (Fig. 2) and experimental results obtained with the UVic LGS-WFS test-bed (Fig. 3).

More details about the analytical model can be found in Ref. [4]. It can be shown that the LGS aberrations induced by a uniform threshold are a combination of focus and spherical aberrations $\left(Z_{11}\right.$, $Z_{37}$, etc.) which scale as the threshold value Thres, the maximum spot elongation $E$ and the sodium profile asymmetry (defined by the function $g$ ). It is interesting to note that a uniform threshold (or a field-stop) would not induce any error with symmetric sodium profiles.

A non-uniform thresholding method, termed "radial thresholding" has been proposed to cancel out most of the LGS aberrations without altering the centroid accuracy [4]. The threshold value is now not uniform over the whole pupil, but is defined independently for each subaperture, proportionally to the maximum of the local spot. The aberration induced by such a thresholding is a pure focus with no spherical aberrations. This focus error is not an issue since it will be corrected by the zoom optics already required to track the sodium layer [1].

\section{LGS-aberration filtering in the frequency domain}

\subsection{Principle}

As the fluctuations of the sodium layer are much slower than the turbulence (typical timescale between 10 and 60s [7]), any residual LGS aberrations can be filtered out in the temporal frequency domain. This filtering technique is applicable to any kind of LGS WFSs and requires:

- a low bandwidth natural guide star (NGS) WFS in addition to the LGS WFS,

- a digital high-pass filter (HPF) on the LGS WFS path.

Basically, the low temporal frequencies are sensed by the NGS-WFS, while the high temporal frequencies are sensed by the LGS-WFS. In other words, we prefer to use the NGS-WFS for sensing the quasi-static aberrations and the slow turbulence, but we still trust (and still need) the LGS WFS 


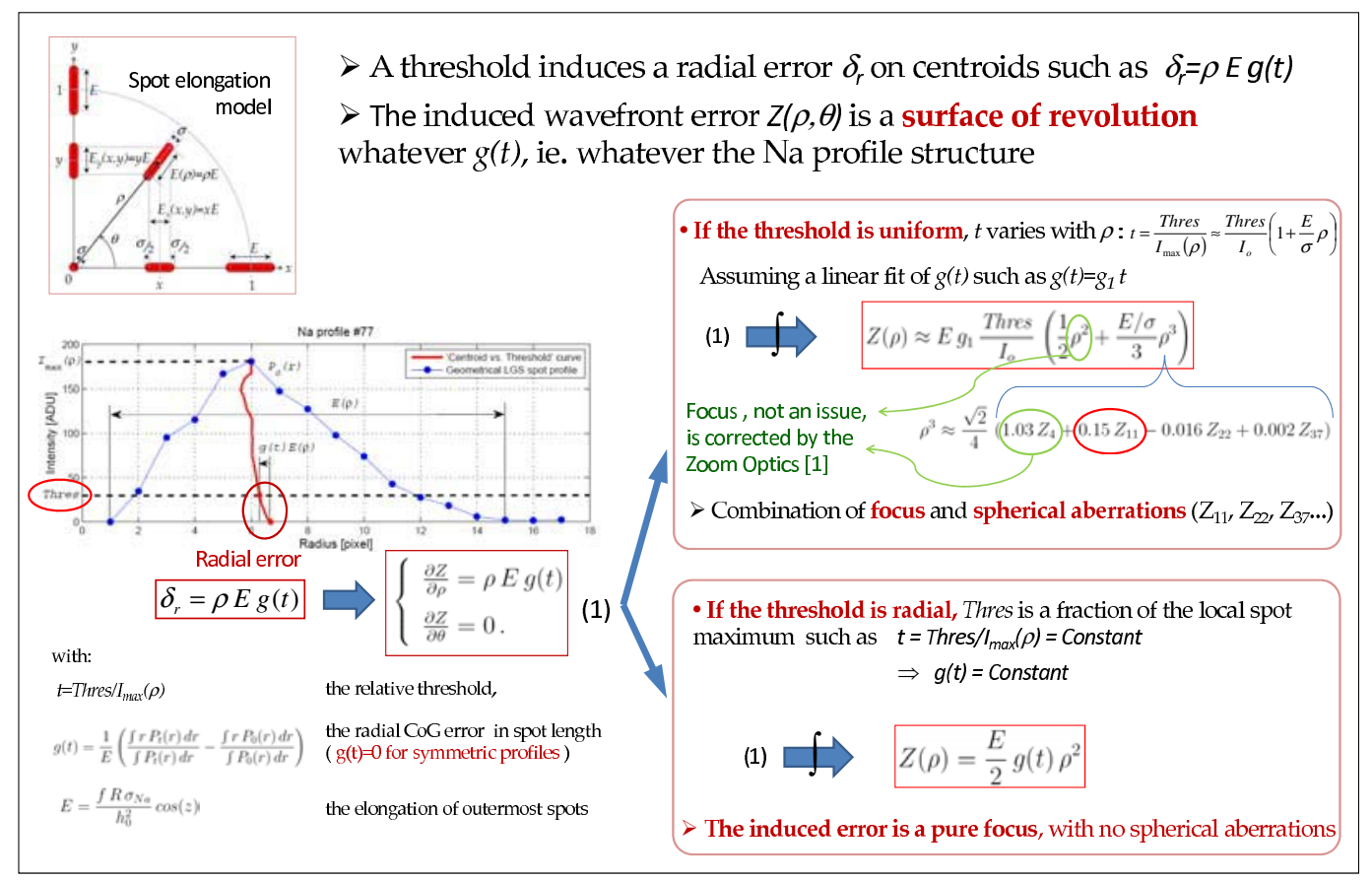

Fig. 2. Modelling of the aberrations induced by thresholding.

to sense the fast turbulence. Ideally, the frame rate of the NGS WFS must be as low as possible not to impact too much the sky coverage.

The low-bandwidth NGS-WFS acts as a low-pass filter (LPF). The HPF has to be defined complementarily to the LPF, such as $H P F=1-L P F$. By doing so, both WFSs complete each other and work in tandem without any conflict. Both WFSs are equivalent to a single WFS sensing all the temporal frequencies. Taking this precaution makes the closed-loop controller more simple (a conventional integrator is enough) and more stable (Fig. 4).

\subsection{Implementation on the UVic LGS-bench}

Figure 5 describes the implementation of the LGS filtering on a real discrete system. This kind of system is usually termed multirate system, as the frame rate of the NGS WFS is different than the frame rate of the LGS WFS.

The HPF consists in subtracting the slopes of a reference image $I_{o}$, which is a periodically-updated time-average of the LGS snapshot images. This HPF already exists in the Matched-Filter [5] and the Correlation [6] algorithms. The reference image is intrinsically required by the algorithms for computing the wavefront slopes, independently to the LGS aberration issue. The update rate of $I_{o}$ must be equal to the NGS WFS frame rate to provide better performance and stability. Ideally both rates should be driven by the same trigger signal as shown in Fig. 5.

\subsection{Bench results}

Figure 6 presents the results obtained in laboratory with the UVic LGS WFS test-bed in closed-loop for the mode $Z_{11}$, the dominant LGS aberration. In addition to the changing sodium profiles, a time series of phase screens is generated by the DM to reproduce the atmospheric turbulence. 


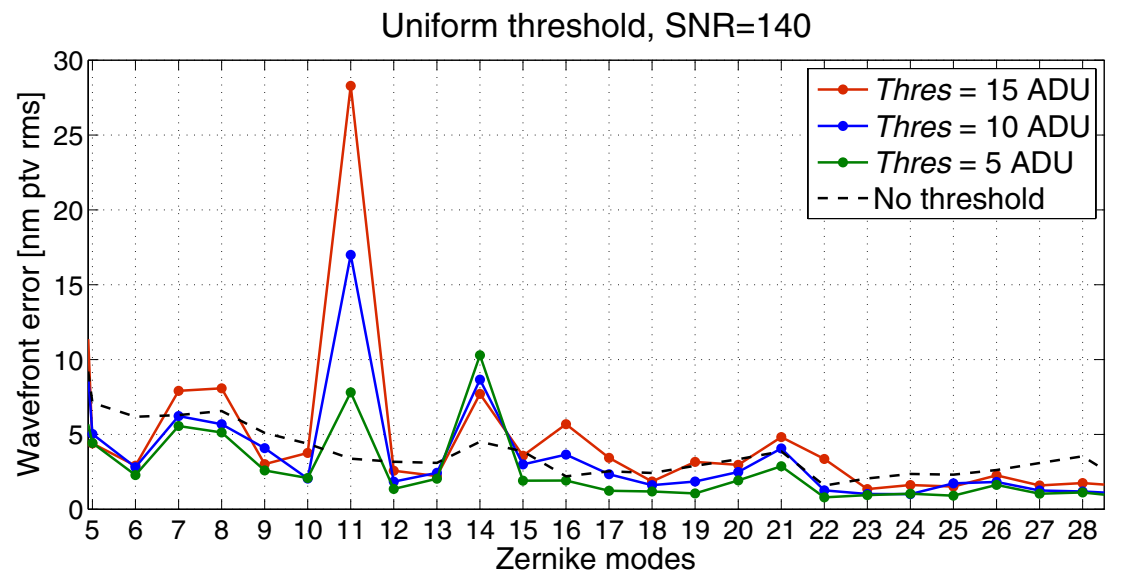

(a)

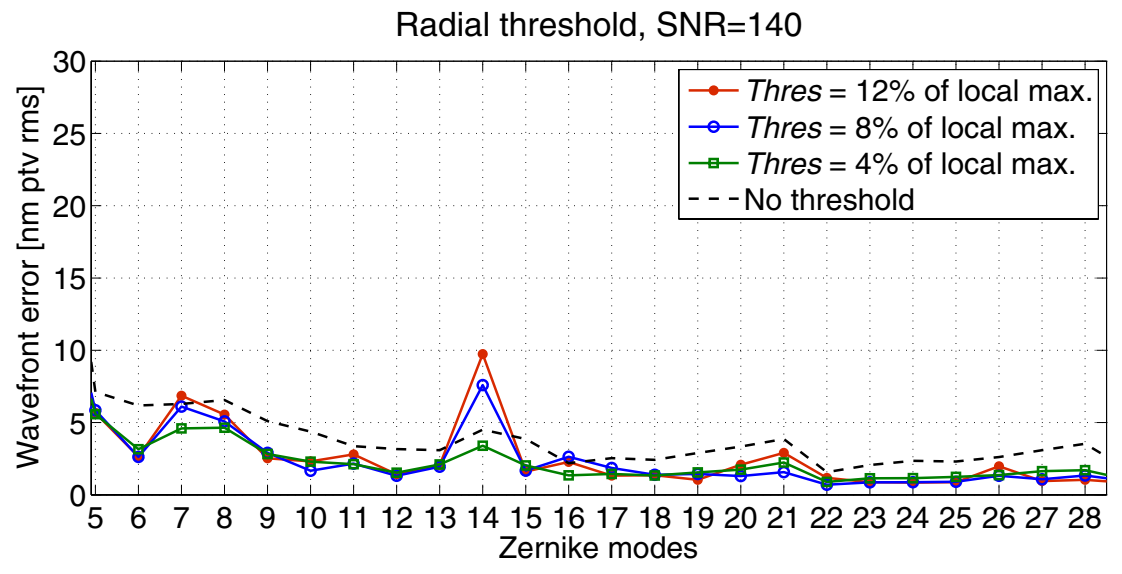

(b)

Fig. 3. LGS aberrations measured on the UVic LGS-WFS test-bed with the 88 sodium profile time series of Fig. 1. (a) With a uniform thresholding, spherical aberration $\left(Z_{11}\right)$ arises proportionally to the threshold value. (b) With a radial thresholding, the $Z_{11}$ mode disappears, only $Z_{14}$, due to the pixel sampling, remains unchanged. The errors arising in coma $\left(Z_{7}\right.$ and $\left.Z_{8}\right)$ are instrumental artefacts of the LGS-bench.

The output wavefront is free of any LGS aberrations after few NGS frames (ie. few seconds). After that time, the closed-loop system corrects the turbulence regardless of the sodium profile fluctuations.

The residual RMS error, obtained with the filter and with the LGS disturbances, is similar to the residual RMS error obtained in the ideal case, with no filter and no LGS disturbances. This result demonstrates that the filtering does not decrease the turbulence correction.

The gain of the NGS WFS has to be reduced to 0.6, relatively to the LGS WFS, in order to provide enough gain margin and make the system more stable.

\section{Conclusion}

The aberrations arising in LGS AO systems due to the sodium layer variations are, beyond the focus, due to instrumental artefact of the LGS wavefront sensing. These aberrations can be mitigated at their source by improving the LGS wavefront sensor itself and the centroiding algorithm. 
(a)
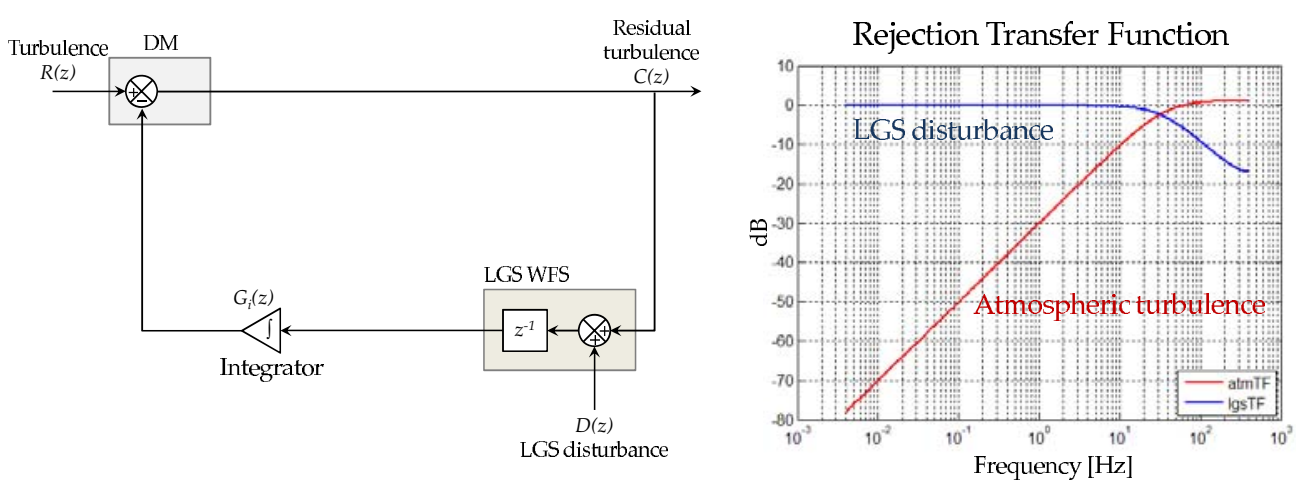

$$
C(z)=\frac{1}{1+z^{-1} G_{i}(z)} R(z)+\frac{-z^{-1} G_{i}(z)}{1+z^{-1} G_{i}(z)} D(z) \quad \text { with } G_{i}(z)=\frac{g}{1-z^{-1}}
$$

(b)

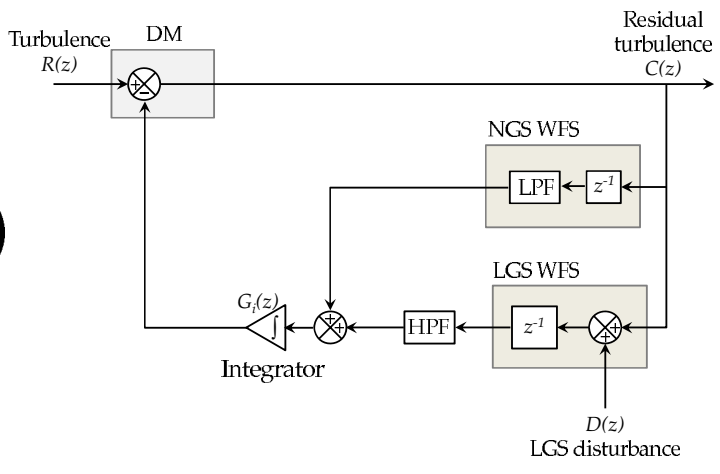

Rejection Transfer Function

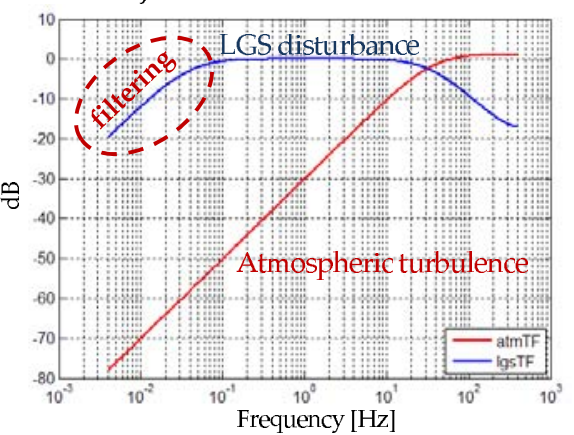

$C(z)=\frac{1}{1+z^{-1} G_{i}(z)(\operatorname{LPF}(z)+\operatorname{HPF}(z))} R(z)+\frac{-z^{-1} G_{i}(z) \operatorname{HPF}(z)}{1+z^{-1} G_{i}(z)(\operatorname{LPF}(z)+\operatorname{HPF}(z))} D(z)$

Fig. 4. Principle of the LGS aberration filtering with a low-bandwidth NGS-WFS. (a) AO system subjected to LGS aberrations. LGS aberrations $D(z)$ are internal feedback disturbances, fully propagated on the science path $C(z)$. (b) LGS aberration filtering: an HPF must be implemented after the LGS WFS. The LPF is the lower frame rate of the NGS-WFS. HPF+LPF should be 1 to act as a pure integrator for turbulence. The rejection transfer functions are plotted for both inputs $R(z)$ and $D(z)$ for a closed-loop system running at $800 \mathrm{~Hz}$.

The radial thresholding presented here is a very simple technique which makes the centre-ofgravity algorithm still well-suited for Extremely Large Telescopes, where the LGS spot elongation is greater. Unlike the matched filter or the correlation centroiding algorithms, the centre-of-gravity is a simple, fast and high dynamic-range algorithm which requires no special calibration processes or reference images, and has no repercussions on the design of the LGS AO system.

If the residual LGS aberrations are still above the error budget, a suitable filter, exploiting the time scale difference between the turbulence and the sodium layer fluctuations, can be implemented too. However, this filter requires a low-bandwidth NGS WFS which may impact the sky coverage and make the whole AO system more complex. For this reason, it seems preferable to work harder on the LGS WFS design first, in order to mitigate as much as possible the LGS aberrations at their source. Lower residual LGS aberrations will have less impact on the sky coverage if a NGS WFS is needed. 

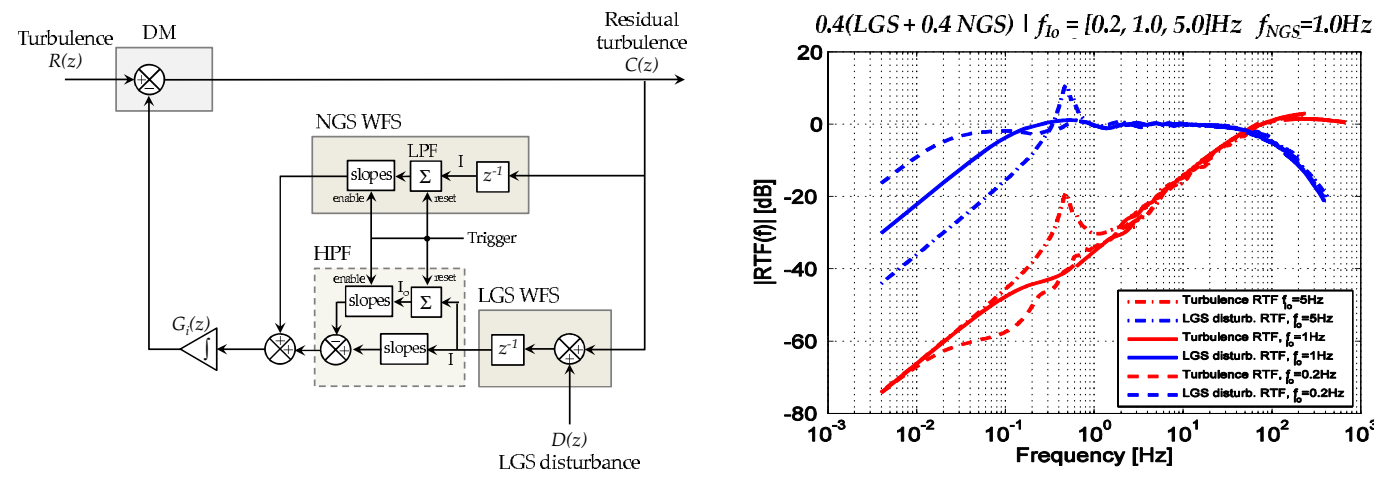

Fig. 5. Implementation of the LGS filtering on a discrete system. The rejection transfer functions are plotted for a NGS WFS running at $f_{N G S}=1 \mathrm{~Hz}$, a LGS WFS running at $800 \mathrm{~Hz}$, and for 3 different update rates of the LGS reference image $I_{o}: f_{I o}=0.2,1$ or $5 \mathrm{~Hz}$. If the $I_{o}$ update rate is equal to the NGS frame rate, the RTF for the turbulence is very similar to the RTF of a pure integrator ( $20 \mathrm{~dB} /$ decade slope). If the $I_{o}$ update rate is faster, the LGS aberration filtering is better but the turbulence correction get worse and the system may be unstable. If the $I_{o}$ update rate is slower than the the NGS WFS frame rate, the turbulence correction is a bit better for the low frequencies, but the filtering of the LGS aberrations is worse.

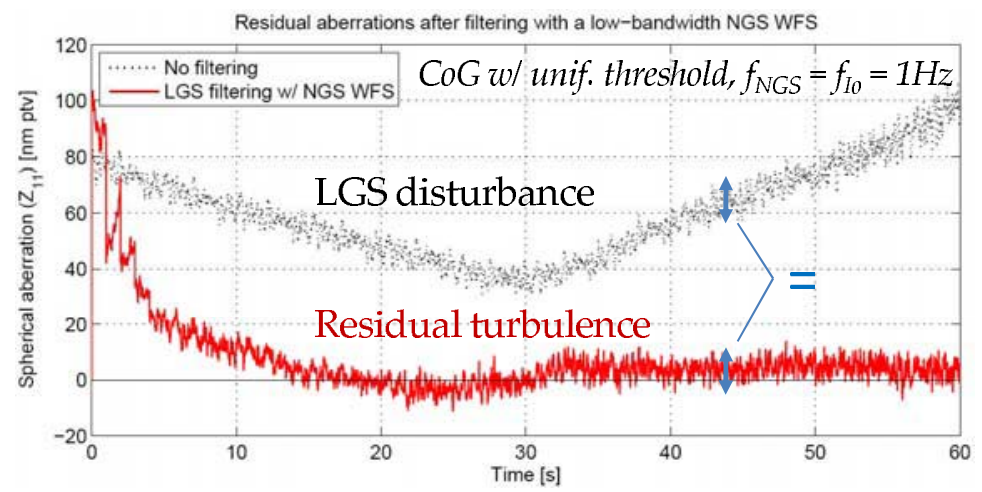

Fig. 6. Results of the LGS filtering obtained with the UVic LGS WFS test-bed in closed-loop for the mode $Z_{11}$. The black dotted curve plots the spherical aberration induced by the LGS disturbance when no filter is applied. The residual RMS error obtained with the filter and with the LGS disturbances, is similar to the residual RMS error obtained in the ideal case, with no filter and no LGS disturbance. The centroiding algorithm used for this figure is the CoG with a uniform thresholding in order to be in a pessimistic case for the LGS aberrations.

\section{References}

1. G. Herriot et al., Proc. SPIE 6272, (2006)

2. R. M. Clare, M. A. van Dam and A. H. Bouchez, Opt. Express , 15, (2007) 4711-4725

3. O. Lardière, R. Conan, C. Bradley, K. Jackson and G. Herriot, Opt. Express, 16, (2008) 5527-5543

4. O. Lardière, R. Conan, C. Bradley, K. Jackson and P. Hampton, MNRAS, 398, (2009), 1461-1467

5. L. Gilles and B. Ellerbroek, Optics Letters, 33, (2008) 1159-1161

6. S. Thomas, S. Adkins, D. Gavel, T. Fusco and V. Michau, MNRAS, 387, (2008) 173-187

7. D. S. Davis, P. Hickson, G. Herriot, and C-Y She, Optics Letters, 31, (2006) 3369-3371 